\title{
Dependence structure analysis of KOSPI and NYSE based on time-varying copula models ${ }^{\dagger}$
}

\author{
Sangyeol Lee ${ }^{1} \cdot$ Byungsoo Kim² \\ ${ }^{12}$ Department of Statistics, Seoul National University \\ Received 10 August 2013, revised 4 September 2013, accepted 30 September 2013
}

\begin{abstract}
In this study, we analyze the dependence structure of KOSPI and NYSE indices based on a two-step estimation procedure. In the first step, we adopt ARMA-GARCH models with Gaussian mixture innovations for marginal processes. In the second step, time-varying copula parameters are estimated. By using these, we measure the dependence between the two returns with Kendall's tau and Spearman's rho. The two dependence measures for various copulas are illustrated.
\end{abstract}

Keywords: ARMA-GARCH models, copula, Gaussian mixture, time-varying copula parameter, two-step estimation.

\section{Introduction}

Copula has been widely used by many researchers when modeling the joint distribution of random vectors with given marginal distributions. Gaussian, Student's $t$, Clayton, Frank, Gumbel and Plackett copulas are common examples: see Nelson (1999), Joe (1997) and Cherubini et al. (2004). Since the copula determines the dependence structure of multivariate distributions, the copula parameter estimation is a very important task. To correctly reflect the dependence changes of asset returns, some authors proposed to use time-varying copula parameters relying on the time-dependent variables or explanatory variables: see Gourieroux and Monfort (1992), Rockinger and Jondeau (2006), Patton (2006b) and Chiou and Tsay (2008), in a spirit similar to the GARCH models of Engle (1982) and Bollerslev (1986).

In this study, we analyze the dependence structure between two market indices, the Korea Composite Stock Price Index (KOSPI) and New York Stock Exchange composite price index (NYSE). To this end, we employ the two-step estimation procedure for the copula based models which introduced by Patton (2006a) with time-varying copula parameters. We apply ARMA-GARCH models with Gaussian mixture innovations (cf. Lee and Lee, 2009, 2011) to the individual returns. As a different approach, we refer to Lee and Lee (2012), who examine stock market co-movement among three China stock markets based on the GARCH-M models.

$\dagger$ This work was supported by the National Research Foundation of Korea (NRF) grant funded by the Korea government (MSIP) No.2012R1A2A2A01046092.

${ }^{1}$ Professor, Department of Statistics, Seoul National University, Seoul 151-742, Korea.

2 Corresponding author: Post doctorial course, Department of Statistics, Seoul National University, Seoul 151-742, Korea. E-mail: bschris1@gmail.com 
This paper is organized as follows. In Section 2, we shortly review the relationship between copula and nonlinear dependence measures and describe the data set. In Section 3, we introduce the two-step estimation procedure. In Section 4, we fit both returns to the ARMA-GARCH models with Gaussian mixture innovations. In Section 5, we measure the dependence between two returns by Kendall's tau and Spearman's rho based on the timevarying copula parameters. Conclusion is provided in Section 6 .

\section{Nonlinear dependence measures and data set}

\subsection{Review of nonlinear dependence measures}

The Pearson's correlation coefficient is the most widely known measure of association between random variables, however it evaluates only the linear relationship. Thus when nonlinear relationship is of main interest, Pearson's coefficient is not suitable. In this study, we apply two nonparametric measures to evaluate the dependence between variables with Kendall's tau and Spearman's rho. For convenience, we concentrate on the bivariate case. These two dependence measures can be expressed using copula such as

$$
\begin{aligned}
\text { Spearman's rho } & :=\rho=12 \int_{0}^{1} \int_{0}^{1} C(u, v) d u d v-3, \\
\text { Kendall's tau } & :=\tau=4 \int_{0}^{1} \int_{0}^{1} C(u, v) d C(u, v)-1 .
\end{aligned}
$$

In particular for the Archimedean copulas, it holds

$$
\tau=1+4 \int_{0}^{1} \frac{\varphi(t)}{\varphi^{\prime}(t)} d t
$$

where $\varphi$ is the generator of the copula $C$.

\subsection{The data set}

We analyze the two stock market indices, KOSPI and NYSE. We compute the daily returns as 100 times the difference of the log of the prices. The daily returns range from December 1, 2008 to May 10, 2013 for both indices. The data set is obtained from finance.yahoo.com and consists of 1079 bivariate time series. Table 2.1 reports the summary statistics for daily returns of the KOSPI and NYSE for the whole period. We can see that both returns have approximately zero mean and kurtosis greater than 3. Figure 2.1 illustrates the daily return series of KOSPI and NYSE. From Figure 2.1, we can notice that the co-movement between two returns is active in the latter half of 2011. Contrastively in the middle of 2010, NYSE series shows high volatilities but KOSPI series doesn't as much as the NYSE.

Table 2.1 Summary statistics for daily returns of the KOSPI and NYSE

\begin{tabular}{cccccccc}
\hline \hline & Mean & Median & S.D. & Min & Max & Skewness & Kurtosis \\
\hline KOSPI & 0.055 & 0.062 & 1.352 & -6.420 & 7.215 & -0.312 & 3.392 \\
NYSE & 0.048 & 0.101 & 1.475 & -9.484 & 7.065 & -0.401 & 4.401 \\
\hline
\end{tabular}



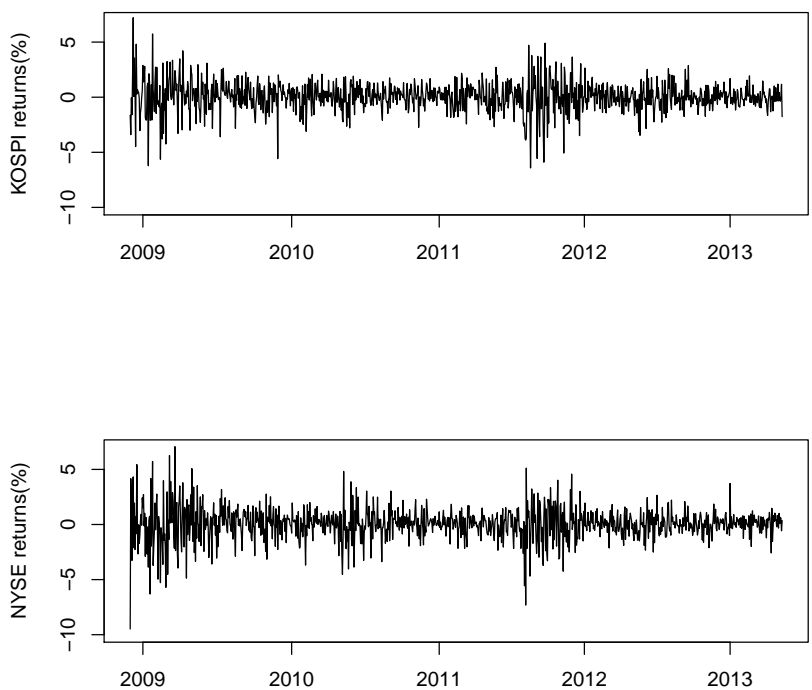

Figure 2.1 Daily return series of the KOSPI and NYSE

\section{Two-step estimation procedure for copula based models}

Although there are many literatures on the estimation theory for copula, most of them assume that the data is independent and identically distributed. However, iid assumption is rejected for almost every financial time series. To overcome this restriction, Patton (2006a) introduced two-step estimation procedure for the copula based models. Assume that there are two return series $\left\{X_{t}, t=1, \ldots, n\right\}$ and $\left\{Y_{t}, t=1, \ldots, n\right\}$. Let the conditional distribution $\left(X_{t}, Y_{t}\right)^{\prime} \mid \mathcal{F}_{t-1}$ be parameterized as $H_{t}(x, y ; \theta)=C\left(F_{t}\left(x ; \theta_{1}\right), G_{t}\left(y ; \theta_{2}\right) ; \alpha\right)$, where $\mathcal{F}_{t-1}$ denotes the information available at time $t-1, F_{t}$ is the conditional distribution function of $X_{t}, G_{t}$ is the conditional distribution function of $Y_{t}, C$ is the copula, $\alpha$ is the copula parameters and $\theta=\left(\theta_{1}^{\prime}, \theta_{2}^{\prime}, \alpha^{\prime}\right)^{\prime}$. Then conditional density of $\left(X_{t}, Y_{t}\right)$ is obtained by

$$
h_{t}(x, y ; \theta)=f_{t}\left(x ; \theta_{1}\right) g_{t}\left(y ; \theta_{2}\right) c\left(F_{t}\left(x ; \theta_{1}\right), G_{t}\left(y ; \theta_{2}\right) ; \alpha\right) \text {, }
$$

where $f_{t}$ is the conditional density of $X_{t}, g_{t}$ is the conditional density of $Y_{t}$ and $c$ is the copula density. According to Patton (2006a), we estimate the parameters $\theta$ by maximizing the $\log$ likelihood function

$$
\hat{\theta}=\underset{\theta}{\operatorname{argmax}}\left[\sum_{t=1}^{n} \log \left(f_{t}\left(x ; \theta_{1}\right)\right)+\log \left(g_{t}\left(y ; \theta_{2}\right)\right)+\log \left(c\left(F_{t}\left(x ; \theta_{1}\right), G_{t}\left(y ; \theta_{2}\right) ; \alpha\right)\right)\right] .
$$

One may utilize the simultaneous estimating method for the parameters in marginal distributions and copula parameter. However as mentioned in Chiou and Tsay (2008), in practice, the number of parameters in objective function may be large and the dependence relation of the copula may involve a convoluted expression of the parameters. In order to overcome this difficulty, we adopt the two-step estimation procedure which is simpler and easier to 
implement. In the first step, we estimate the parameters in marginal distributions. See the Section 4 for details. In the second step, we estimate the copula parameter based on the estimated parameters in the first step, that is

$$
\hat{\alpha}=\underset{\alpha}{\operatorname{argmax}} \sum_{t=1}^{n} \log \left(c\left(F_{t}\left(x ; \hat{\theta}_{1}\right), G_{t}\left(y ; \hat{\theta}_{2}\right) ; \alpha\right)\right) .
$$

Details are presented in Section 5. Patton (2006a) showed that the two-step estimator is asymptotically normally distributed under some regularity conditions. Since the Kendall's tau and Spearman's rho depend only on the copula parameters, we can analyze the dependence between two returns with the estimated copula parameters.

\section{Marginal models and parameter estimation}

In this section, we employ the $\operatorname{ARMA}(p, q)-\operatorname{GARCH}(1,1)$ models for the individual returns of KOSPI and NYSE.

\section{1. $\operatorname{ARMA}(p, q)-\operatorname{GARCH}(\mathrm{r}, \mathrm{s})$ model with Gaussian mixture innovations}

For the individual returns $r_{t}, t=1, \ldots, n$, let us consider the $\operatorname{ARMA}(p, q)-\operatorname{GARCH}(\mathrm{r}, \mathrm{s})$ model

$$
\begin{aligned}
r_{t} & =c_{0}+\sum_{i=1}^{p} c_{i} r_{t-i}+\epsilon_{t}+\sum_{j=1}^{q} d_{j} \epsilon_{t-j} \\
\epsilon_{t} & =\sigma_{t} z_{t} \\
\sigma_{t}^{2} & =\omega+\sum_{i=1}^{s} a_{i} \epsilon_{t-i}^{2}+\sum_{j=1}^{r} b_{j} \sigma_{t-j}^{2},
\end{aligned}
$$

where $c_{i}(i=0, \ldots, p), d_{j}(j=1, \ldots, q) \in \mathbb{R}, \omega>0, a_{i}(i=1, \ldots, s), b_{j}(j=1, \ldots, r) \geq$ $0,1-\sum_{i=1}^{p} c_{i} z^{i} \neq 0$ and $1+\sum_{j=1}^{q} d_{j} z^{j} \neq 0$ for $|z| \leq 1$. Further, we assume that $\sum_{i=1}^{s} a_{i}+$ $\sum_{j=1}^{r} b_{j}<1$ for the second order stationary of GARCH model. Chiou and Tsay (2008) also considered the ARMA-GARCH models with a constant conditional mean for marginal returns. However, although their data shows leptokurtic property, they assumed that the innovation $z_{t}$ is a sequence of independent and identically distributed random variables with Gaussian distribution. From the past experience, it is well known that the Gaussian assumption is not satisfied frequently, especially in the financial field. To cope with such a defect, in this study we assume that $z_{t}$ is a sequence of independent and identically distributed random variables with $m$-component Gaussian mixture, $\operatorname{say} \operatorname{NM}\left(\pi_{k}, \mu_{k}, \zeta_{k}, k=\right.$ $1,2, \ldots m)$, density defined as

$$
\psi(y)=\sum_{k=1}^{m} \pi_{k} \phi\left(y ; \mu_{k}, \zeta_{k}\right),
$$

where $\phi\left(\cdot ; \mu_{k}, \zeta_{k}\right)$ is the Gaussian density with mean $\mu_{k}$ and standard deviation $\zeta_{k}, \sum_{k=1}^{m} \pi_{k}=$ 1, $\sum_{k=1}^{m} \pi_{k} \mu_{k}=0$ and $\sum_{k=1}^{m} \pi_{k}\left(\mu_{k}^{2}+\zeta_{k}^{2}\right)=1$. The last two assumptions imply that 
$E\left(z_{t}\right)=0$ and $\operatorname{Var}\left(z_{t}\right)=1$. In order to estimate the ARMA-GARCH model and mixture parameters, we follow the estimation procedure described below. Throughout this study, we choose $r_{1}$ as the initial values for $r_{0}, . ., r_{1-p}, \epsilon_{0}, \ldots, \epsilon_{1-\max \{q, s\}}$ and $\sigma_{0}, \ldots \sigma_{1-r}$.

1. Obtain the Gaussian QMLE for the parameters $c_{i}(i=0, \ldots, p), d_{j}(j=1, \ldots, q), \omega$, $a_{i}(i=1, \ldots, s)$ and $b_{j}(j=1, \ldots, r)$. Francq and Zakoian (2004) showed that the Gaussian QMLE is consistent and asymptotically normally distributed under regularity conditions.

2. Obtain the ARMA-GARCH residuals based on the QMLE

$$
\tilde{z}_{t}=\frac{\tilde{\epsilon}_{t}}{\tilde{\sigma}_{t}}, t=1, \ldots, n,
$$

where $\tilde{\epsilon}_{t}$ and $\tilde{\sigma}_{t}^{2}, t=1, \ldots, n$ are defined recursively by $\tilde{\epsilon}_{t}=r_{t}-\hat{c}_{0}-\sum_{i=1}^{p} \hat{c}_{i} r_{t-i}-\sum_{j=1}^{q} \hat{d}_{j} \tilde{\epsilon}_{t-j}$ and $\tilde{\sigma}_{t}^{2}=\hat{\omega}+\sum_{i=1}^{s} \hat{a}_{i} \tilde{\epsilon}_{t-i}^{2}+\sum_{j=1}^{r} \hat{b}_{j} \tilde{\sigma}_{t-j}^{2}$.

3. By using ARMA-GARCH residuals, mixture parameters are estimated by maximizing the log likelihood function

$$
\frac{1}{n} \sum_{t=1}^{n} \log \left(\psi\left(\tilde{z}_{t}\right)\right)
$$

We use the EM algorithm to find the solutions. Given the current parameter $\eta^{(l)}=\left(\pi_{k}^{(l)}, \mu_{k}^{(l)}\right.$, $\left.\zeta_{k}^{(l)}\right)$, the EM algorithm for the Gaussian mixture model interates as follows

$$
\begin{aligned}
\pi_{k}^{(l+1)} & =\frac{1}{n} \sum_{t=1}^{n} \beta\left(k \mid \tilde{z}_{t} ; \eta^{(l)}\right) \\
\mu_{k}^{(l+1)} & =\frac{\sum_{t=1}^{n} \tilde{z}_{t} \beta\left(k \mid \tilde{z}_{t} ; \eta^{(l)}\right)}{\sum_{t=1}^{n} \beta\left(k \mid \tilde{z}_{t} ; \eta^{(l)}\right)} \\
\sigma_{k}^{2(l+1)} & =\frac{\sum_{t=1}^{n} \tilde{z}_{t}^{2} \beta\left(k \mid \tilde{z}_{t} ; \eta^{(l)}\right)}{\sum_{t=1}^{n} \beta\left(k \mid \tilde{z}_{t} ; \eta^{(l)}\right)}-\left(\mu_{k}^{(l+1)}\right)^{2},
\end{aligned}
$$

where $\beta\left(k \mid \tilde{z}_{t} ; \eta^{(l)}\right)=\pi_{k}^{(l)} \phi\left(\tilde{z}_{t} ; \mu_{k}^{(l)}, \zeta_{k}^{(l)}\right) / \sum_{j=1}^{m} \pi_{j}^{(l)} \phi\left(\tilde{z}_{t} ; \mu_{j}^{(l)}, \zeta_{j}^{(l)}\right)$, for $k=1, \ldots . m$. The quasi clustering technique in Woodward et al. (1984) is employed for selecting an initial value. Note that occasionally, the estimators obtained from EM algorithm may not satisfy the assumptions imposed on the mixture parameters. Let, $\hat{\eta}^{e m}=\left(\hat{\pi}_{k}^{e m}, \hat{\mu}_{k}^{e m}, \hat{\zeta}_{k}^{e m}, k=1, \ldots, m\right)^{\prime}$ denote the estimators from EM algorithm. According to Lee and Lee (2011), we can use a modified version of $\hat{\eta}^{e m}$ to resolve this problem,

$$
\hat{\eta}^{*}=\left(\hat{\pi}_{k}^{*}, \hat{\mu}_{k}^{*}, \hat{\zeta}_{k}^{*}, k=1, \ldots, m\right)^{\prime}:=\left(\hat{\pi}_{k}^{e m}, \frac{\hat{\mu}_{k}^{e m}-\hat{\mu}}{\hat{\zeta}}, \frac{\hat{\zeta}_{k}^{e m}}{\hat{\zeta}}, k=1, \ldots, m\right)^{\prime},
$$

where $\hat{\mu}=\sum_{k=1}^{m} \hat{\pi}_{k}^{e m} \hat{\mu}_{k}^{e m}$ and $\hat{\zeta}^{2}=\sum_{k=1}^{m} \hat{\pi}_{k}^{e m}\left(\left(\hat{\mu}_{k}^{e m}-\hat{\mu}\right)^{2}+\left(\hat{\zeta}_{k}^{e m}\right)^{2}\right)$. Lee and Lee $(2011)$ demonstrated that $\hat{\eta}^{e m}$ is a consistent estimator and so is the modified estimator $\hat{\eta}^{*}$.

4. Using the ARMA-GARCH and mixture parameter estimators, conditional distribution function of $r_{t}$ can be estimated (called $F_{t}\left(\cdot ; \hat{\theta}_{1}\right)$ and $G_{t}\left(\cdot ; \hat{\theta}_{2}\right)$ in Section 3 ) as the distribution function of $\operatorname{NM}\left(\hat{\pi}_{k}^{*}, \hat{c}_{0}+\sum_{i=1}^{p} \hat{c}_{i} r_{t-i}+\sum_{j=1}^{q} \hat{d}_{j} \tilde{\epsilon}_{t-j}+\tilde{\sigma}_{t} \hat{\mu}_{k}^{*}, \tilde{\sigma}_{t} \hat{\zeta}_{k}^{*}, k=1,2, \ldots m\right)$. 


\subsection{Application to the KOSPI and NYSE}

Figure 4.1 depicts the autocorrelation function $(\mathrm{ACF})$ and partial autocorrelation function (PACF) of the KOSPI and NYSE. Since the ACF and PACF are modest in size except for the PACF with lag 1 of NYSE, we choose an AR(1) model for the conditional mean of ARMA-GARCH models for both returns. Table 4.1 shows the Gaussian QMLE for the AR(1)-GARCH(1,1) model parameters. We perform the Jarque-Bera and Ljung-Box tests for the diagnostics of fitted model based on the standardized residuals. Table 4.2 reports the p-values of each tests. In Table $4.2, Q(h)$ and $Q^{2}(h)$ denote the Ljung-Box tests for the standardized residuals and squared standardized residuals with lag $h$, respectively. From the results of Jarque-Bera test, we can speculate that the Gaussian assumption on $z_{t}$ may be inadequate for our data. The results of Ljung-Box tests inform that the standardized residuals do not have autocorrelations and ARCH effects at the significance level 0.05. Thus, both the conditional mean and variance equations are proper in describing the first two moments of the data. In this study, we use the two component Gaussian mixture model. The modified version of mixture parameter estimators, denoted by $\hat{\eta}^{*}$ in Section 4.1, is presented in Table 4.3. In Table 4.3, standard errors are obtained by bootstrap.

Remark 4.1 We remark that the conditional mean modeling with a constant and higher orders has been examined. However, conditional mean modeling did not strongly affect the results. Hence, we only consider the AR(1) model for KOSPI and NYSE returns.

Series KOSPI

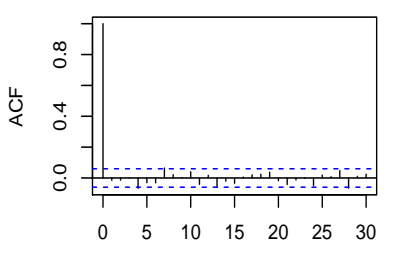

Lag

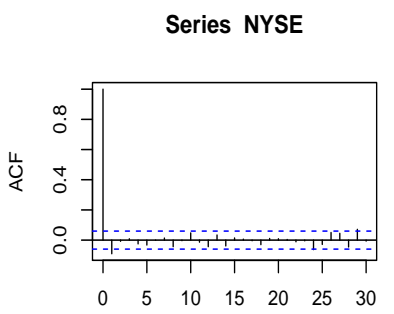

Lag
Series KOSPI

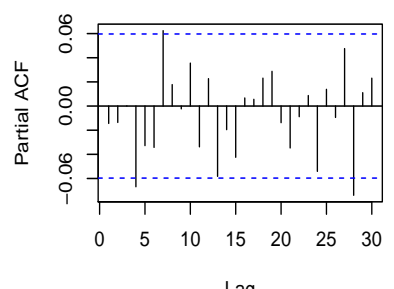

Lag

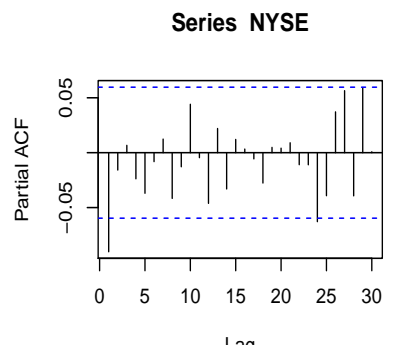

Lag

Figure 4.1 ACF and PACF of the KOSPI and NYSE 
Table 4.1 Gaussian QMLE for the AR(1)-GARCH(1,1) model parameters: The numbers in parentheses are standard errors and the symbols *, $* *,{ }^{* *}$ indicates significance at the level $10 \%, 5 \%, 1 \%$

\begin{tabular}{cccccc}
\hline \hline & $\hat{c}_{0}$ & $\hat{c}_{1}$ & $\hat{\omega}$ & $\hat{a}$ & $\hat{b}$ \\
\hline KOSPI & $0.058(0.033)^{*}$ & $-0.034(0.026)$ & $0.018(0.009)^{* *}$ & $0.067(0.016)^{* * *}$ & $0.922(0.017)^{* * *}$ \\
NYSE & $0.087(0.034)^{*}$ & $-0.053(0.027)^{* *}$ & $0.031(0.012)^{* * *}$ & $0.093(0.024)^{* * *}$ & $0.890(0.024)^{* * *}$ \\
\hline
\end{tabular}

Table 4.2 P-values for the Jarque-Bera and Ljung-Box tests

\begin{tabular}{ccc}
\hline \hline & KOSPI & NYSE \\
\hline Jarque-Bera & $<2.2 \times 10^{-16}$ & $<2.2 \times 10^{-16}$ \\
$Q(10)$ & 0.7452 & 0.9526 \\
$Q(20)$ & 0.4580 & 0.994 \\
$Q(30)$ & 0.4692 & 0.9625 \\
$Q^{2}(10)$ & 0.5643 & 0.0968 \\
$Q^{2}(20)$ & 0.5396 & 0.4568 \\
$Q^{2}(30)$ & 0.6738 & 0.8227 \\
\hline
\end{tabular}

Table 4.3 Modified version of the Gaussian mixture parameter estimators: The numbers in parentheses are standard errors

\begin{tabular}{cccccc}
\hline \hline & $\hat{\pi}_{1}^{*}$ & $\hat{\mu}_{1}^{*}$ & $\hat{\mu}_{2}^{*}$ & $\hat{\zeta}_{1}^{*}$ & $\hat{\zeta}_{2}^{*}$ \\
\hline KOSPI & $0.263(0.032)$ & $-0.494(0.068)$ & $0.176(0.017)$ & $1.315(0.032)$ & $0.788(0.016)$ \\
NYSE & $0.454(0.025)$ & $-0.206(0.029)$ & $0.172(0.014)$ & $1.295(0.024)$ & $0.610(0.017)$ \\
\hline
\end{tabular}

\section{Time-varying copula parameter estimation and dependence measures}

In this study, we follow the specifications of Chiou and Tsay (2008) for time-varying copula parameters to reveal the dependence pattern between KOSPI and NYSE returns. We consider four copulas in this paper. Chiou and Tsay (2008) selected the Plackett and Frank copulas since they enjoy some nice properties. The density of two copulas are flexible for the maginal processes and dependence between two marginal processes is represented by a single parameter. Further, both copulas are comprehensive. A copula which interpolates the positive dependence, negative dependence and independence by varying the parameter is called comprehensive. We consider the Plackett and Frank copulas following Chiou and Tasy (2008) and the most commonly used copulas, Guassian and Student's $t$ copulas in addition. Guassian and Student's $t$ copulas are also comprehensive and Student's $t$ copula can generate joint tail dependence.

Below, we provide the copula functions, density functions and related dependence measures of four copulas.

1. Plackett copula

Plackett copula for $\alpha>0, \alpha \neq 1$ is

$C(u, v ; \alpha)=\frac{1}{2(\alpha-1)}\left[1+(\alpha-1)(u+v)-\sqrt{\{1+(\alpha-1)(u+v)\}^{2}-4 u v \alpha(\alpha-1)}\right]$,

and for $\alpha=1$,

$$
C(u, v ; 1)=u v .
$$


It has the density of the form

$$
c(u, v ; \alpha)=\frac{\alpha\{1+(u-2 u v+v)(\alpha-1)\}}{\left[\{1+(\alpha-1)(u+v)\}^{2}-4 u v \alpha(\alpha-1)\right]^{3 / 2}} .
$$

Plackett copula parameter $\alpha$ is related to the Spearman's rho as

$$
\begin{aligned}
\rho & =\frac{\alpha+1}{\alpha-1}-\frac{2 \alpha \log (\alpha)}{(\alpha-1)^{2}} & \text { for } \alpha \neq 1 \\
& =0 & \text { for } \alpha=1 .
\end{aligned}
$$

2. Frank copula

Frank copula is

$$
C(u, v ; \alpha)=-\frac{1}{\alpha} \log \left[1+\frac{\left(e^{-\alpha u}-1\right)\left(e^{-\alpha v}-1\right)}{e^{-\alpha}-1}\right],
$$

where $\alpha \neq 0$. The density function of the Frank copula is

$$
c(u, v ; \alpha)=\frac{-\alpha\left(e^{-\alpha}-1\right) e^{-\alpha(u+v)}}{\left\{\left(e^{-\alpha u}-1\right)\left(e^{-\alpha v}-1\right)+\left(e^{-\alpha}-1\right)\right\}^{2}} .
$$

Frank copula parameter $\alpha$ is related to the Spearman's rho and Kendall's tau as

$$
\begin{aligned}
\rho & =1-\frac{12}{\alpha}\left[D_{1}(\alpha)-D_{2}(\alpha)\right], \\
\tau & =1+\frac{4}{\alpha}\left[D_{1}(\alpha)-1\right],
\end{aligned}
$$

where $D_{k}(x)$ denotes the "Debye" function

$$
D_{k}(x)=\frac{k}{x^{k}} \int_{0}^{x} \frac{t^{k}}{e^{t}-1} d t, k=1,2 .
$$

3. Gaussian copula

Let the correlation be $\alpha$ between two marginal preocesses. Then, Gaussian copula is

$$
C(u, v ; \alpha)=\int_{-\infty}^{\Phi^{-1}(u)} \int_{-\infty}^{\Phi^{-1}(v)} \frac{1}{2 \pi \sqrt{1-\alpha^{2}}} \exp \left(-\frac{s^{2}-2 \alpha s t+t^{2}}{2\left(1-\alpha^{2}\right)}\right) d s d t
$$

where $\Phi$ denotes the univariate standard Gaussian distribution function and $-1<\alpha<1$. The density of the Gaussian copula is

$$
c(u, v ; \alpha)=\frac{1}{\sqrt{1-\alpha^{2}}} \exp \left(\frac{2 \alpha \Phi^{-1}(u) \Phi^{-1}(v)-\alpha^{2}\left(\Phi^{-1}(u)^{2}+\Phi^{-1}(v)^{2}\right)}{2\left(1-\alpha^{2}\right) .}\right) .
$$

Gaussian copula parameter $\alpha$ is associated with the Spearman's rho and Kendall's tau as

$$
\begin{aligned}
\rho & =\frac{6}{\pi} \arcsin \left(\frac{\alpha}{2}\right), \\
\tau & =\frac{2}{\pi} \arcsin (\alpha) .
\end{aligned}
$$




\section{Student's $t$ copula}

Student's $t$ copula has two parameters, correlation $\alpha$ and degree of freedom $\nu$. Then, Student's $t$ copula is formulated by

$$
C(u, v ; \alpha, \nu)=\int_{-\infty}^{T_{\nu}^{-1}(u)} \int_{-\infty}^{T_{\nu}^{-1}(v)} \frac{1}{2 \pi \sqrt{1-\alpha^{2}}}\left(1+\frac{s^{2}-2 \alpha s t+t^{2}}{\nu\left(1-\alpha^{2}\right)}\right)^{-(\nu+2) / 2} d s d t
$$

where $T_{\nu}$ denotes the univariate Student's $t$ distribution function with degree of freedom $\nu>0$ and $-1<\alpha<1$. The density of the Student's $t$ copula is

$c(u, v ; \alpha, \nu)=\frac{1}{\sqrt{1-\alpha^{2}}} \frac{\Gamma((v+2) / 2) \Gamma(\nu / 2)}{\Gamma((\nu+1) / 2)^{2}} \frac{\left(1+x_{u}^{2} / \nu\right)^{(\nu+1) / 2}\left(1+x_{v}^{2} / \nu\right)^{(\nu+1) / 2}}{\left\{1+\left(x_{u}^{2}+x_{v}^{2}-2 \alpha x_{u} x_{v}\right) / \nu\left(1-\alpha^{2}\right)\right\}^{(\nu+2) / 2}}$,

where $x_{u}=T_{\nu}^{-1}(u)$ and $x_{v}=T_{\nu}^{-1}(v)$. Note that the Kendall's tau is influenced only by $\alpha$ such as

$$
\tau=\frac{2}{\pi} \arcsin (\alpha)
$$

According to Chiou and Tsay (2008), to emphasize that the dependence may vary with the volatility of marginal processes, we specify the time-varying copula parameter as

$$
\alpha_{t} \mid \mathcal{F}_{t-1}=\Lambda\left(d_{1}+d_{2} \sigma_{t}^{K O S P I}+d_{3} \sigma_{t}^{N Y S E}+d_{4} \sqrt{\sigma_{t}^{K O S P I} \sigma_{t}^{N Y S E}}\right),
$$

where $\sigma_{t}^{K O S P I}$ and $\sigma_{t}^{N Y S E}$ are the volatilities of the KOSPI and NYSE, respectively. Note that many specifications are available to model the copula parameter. See also the studies introduced in Section 1. To ensure the constraints that imposed on $\alpha$ of four copulas are satisfied, we adopt the function $\Lambda$ distinctly as follows:

1. Plackett copula: $\Lambda(x)=e^{x}$,

2. Frank copula: $\Lambda(x)=x$,

3. Normal and Student's $t$ copulas: $\Lambda(x)=\left(1-e^{-x}\right) /\left(1+e^{-x}\right)$.

Note that the above three expressions for $\Lambda(x)$ have common characteristic that as $x$ approaches $\infty,-\infty$ and 0 , corresponding copulas imply positive dependence, negative dependence and independence, respectively. To obtain the estimators for $d_{1}, d_{2}, d_{3}$ and $d_{4}$, we substitute $\alpha$ to $\alpha_{t}$ in (3.1). Then we can estimate the time-varying copula parameters as

1. Plackett copula:

$$
\hat{\alpha}_{t} \mid \mathcal{F}_{t-1}=\exp \left(0.687+1.812 \sigma_{t}^{K O S P I}+0.776 \sigma_{t}^{N Y S E}-2.432 \sqrt{\sigma_{t}^{K O S P I} \sigma_{t}^{N Y S E}}\right),
$$

2. Frank copula:

$$
\hat{\alpha}_{t} \mid \mathcal{F}_{t-1}=1.320+4.173 \sigma_{t}^{K O S P I}+1.814 \sigma_{t}^{N Y S E}-5.619 \sqrt{\sigma_{t}^{K O S P I} \sigma_{t}^{N Y S E}},
$$

3. Gaussian copula:

$$
\hat{\alpha}_{t} \mid \mathcal{F}_{t-1}=\frac{1-\exp \left(-0.462-1.098 \sigma_{t}^{K O S P I}-0.378 \sigma_{t}^{N Y S E}+1.334 \sqrt{\sigma_{t}^{K O S P I} \sigma_{t}^{N Y S E}}\right)}{1+\exp \left(-0.462-1.098 \sigma_{t}^{K O S P I}-0.378 \sigma_{t}^{N Y S E}+1.334 \sqrt{\sigma_{t}^{K O S P I} \sigma_{t}^{N Y S E}}\right)},
$$


4. Student's $t$ copula:

$\hat{\alpha}_{t} \mid \mathcal{F}_{t-1}=\frac{1-\exp \left(-0.452-1.179 \sigma_{t}^{K O S P I}-0.413 \sigma_{t}^{N Y S E}+1.450 \sqrt{\sigma_{t}^{K O S P I} \sigma_{t}^{N Y S E}}\right)}{1+\exp \left(-0.452-1.179 \sigma_{t}^{K O S P I}-0.413 \sigma_{t}^{N Y S E}+1.450 \sqrt{\sigma_{t}^{K O S P I} \sigma_{t}^{N Y S E}}\right)}$.

In Figures 5.1, 5.2 and 5.3, the dashed horizontal lines represent the Spearman's rho and Kendall's tau when the copula parameters are time-invariant. We can easily see that we may lose a great deal of informations about the dependence changes when we assume that the copula parameters are time-invariant. Figure 5.1 compares Spearman's rho and Kendall's tau under the Frank and Gaussaian copulas based on the time-varying copula parameters. Although the two dependence measures are different in size, but they show similar patterns. Figure 5.2 illustrates Spearman's rho under the Plackett, Frank and Gaussian copulas and Figure 5.3 depicts Kendall's tau under the Frank, Gaussian and Student's $t$ copulas based on the time-varying copula parameters. In Figures 5.2 and 5.3, the dependence patterns are almost same among the copulas which indicates that the dependence measures are not sensitive to the choice of copulas in our data. Dependence is the strongest in September 28, 2011 and weakest in December 15, 2008. Note that as anticipated from Figure 2.1, dependence measures show high values in the latter half of 2011 and low values in the middle of 2010. Actually in the latter half of 2011, the world market indices had been faltered together due to the economic crisis originated from the Europe and U.S. These facts support that the two-step estimation procedure with time-varying copula parameter reflects well the actual dependence structure of data. Therefore, we think it would be a promising tool to identify the dependence structure along with the time.
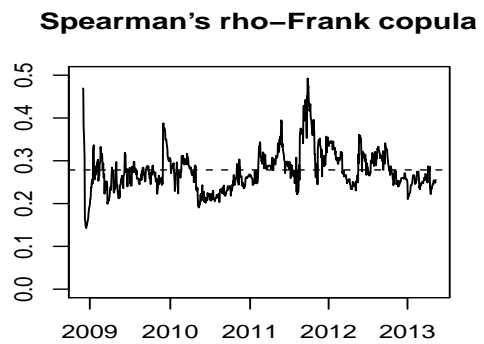

Spearman's rho-Gaussian copula

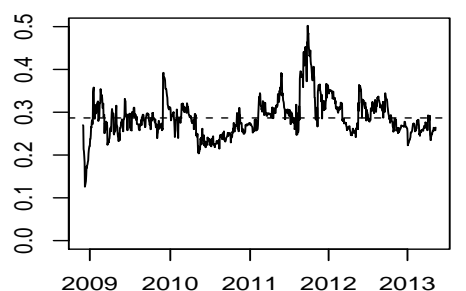

Kendall's tau-Frank copula

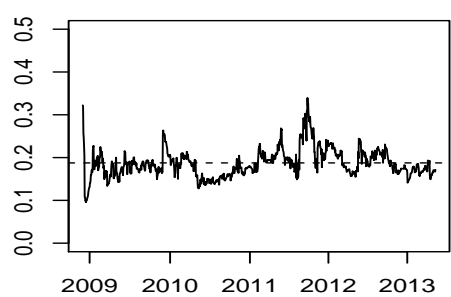

Kendall's tau-Gaussian copula

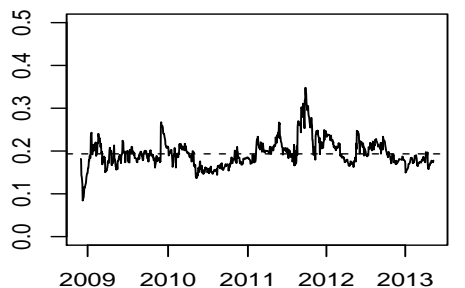

Figure 5.1 Spearman's rho and Kendall's tau between KOSPI and NYSE returns under the Frank and Gaussian copulas based on the time-varying copula parameters: Dashed lines represent the Spearman's rho and Kendall's tau when the copula parameters are time-invariant. 

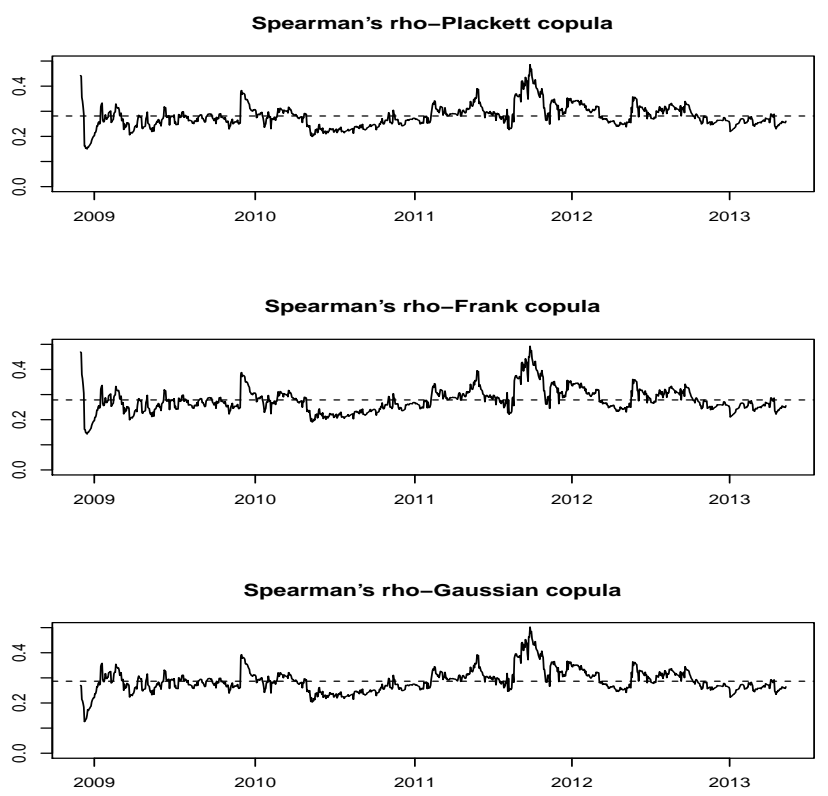

Figure 5.2 Spearman's rho between KOSPI and NYSE returns under the Plackett, Frank and Gaussian copulas based on the time-varying copula parameters: Dashed lines represent the Spearman's rho when the copula parameters are time-invariant.
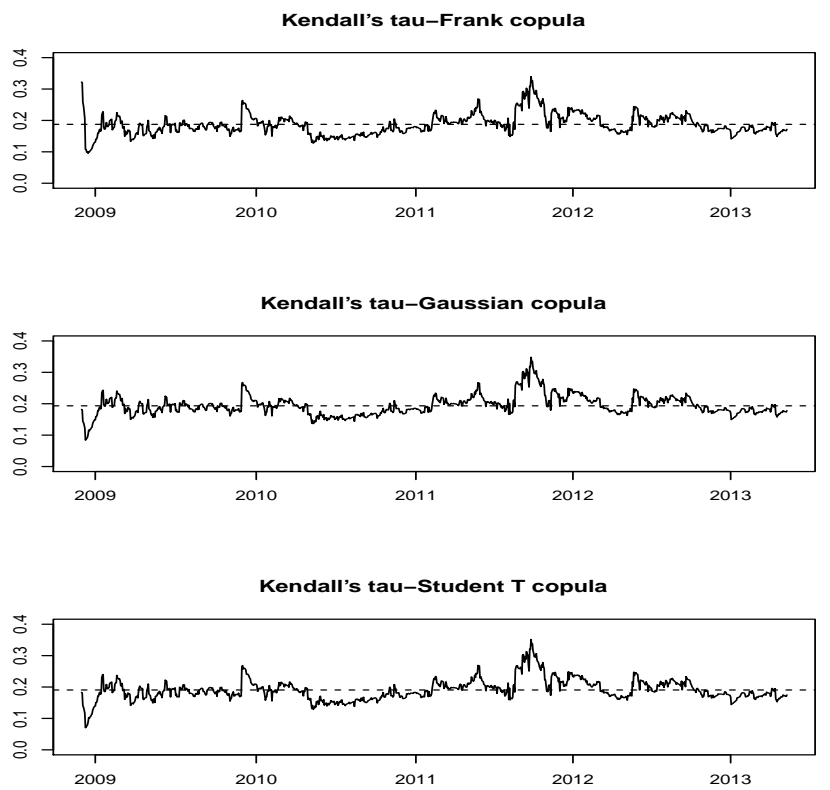

Figure 5.3 Kendall's tau between KOSPI and NYSE returns under the Frank, Gaussian and Student's $t$ copulas based on the time-varying copula parameters: Dashed lines represent the Kendall's tau when the copula parameters are time-invariant. 


\section{Conclusion}

In this paper, we analyzed the dependence structure between two stock market indices KOSPI and NYSE by using the Patton's (2006a) two-step estimation procedure for copula based models. In the first step, $\operatorname{AR}(1)-\operatorname{GARCH}(1,1)$ with Gaussian mixture innovation models were employed for marginal returns. We adopted two component Gaussian mixture distributions, since it conventionally performs better than Gaussian ARMA-GARCH models when data shows leptokurtic property. Selection of mixture order can be made by comparing the AIC and BIC. Further, we refer to Lee and Lee (2008) for the robust estimation of mixture order. Diagnostic results validated the selected models. In the second step, we considered four copulas with time-varying copula parameter which vary with marginal volatilities. Time-varying copula parameter can be specified diversely, for example, Patton (2006a) used the conditional copula parameter in a manner similar to that used by a GARCH model to capture time-varying volatility. Dependence was measured by Kendall's tau and Spearman's rho. As a result, we found that the two-step estimation procedure with timevarying copula parameter is very valuable when we want to know the dependence pattern over the time.

\section{References}

Bollerslev, T. (1986). Generalized autoregressive conditional heteroskedasticity. Journal of Econometrics, 31, 307-327.

Cherubini, U., Luciano, E. and Vecchiato, W. (2004). Copula methods in finance, Wiley, West Sussex.

Chiou, S. C. and Tsay, R. S. (2008). A copula-based approach to option pricing and risk assessment. Journal of Data Science, 6, 273-301.

Engle, R. F. (1982). Autoregressive conditional heteroskedasticity with estimates of the variance of United Kingdom inflation. Econometrica, 50, 987-1008.

Francq, C. and Zakoian, J. (2004). Maximum likelihood estimation of pure GARCH and ARMA-GARCH processes. Bernoulli, 10, 605-637.

Gourieroux, G. and Monfort, A. (1992). Qualitative threshold ARCH models. Journal of Econometrics, 52 , 159-199.

Joe, H. (1997). Multivariate models and dependence concepts, Chapman and Hall, London.

Lee, S. and Lee, E. (2012). A study on the information transfer effect among the China stock markets. Journal of the Korean Data \& Information Science Society, 23, 1075-1084.

Lee, S. and Lee, T. (2008). Robust estimation for the order of finite mixture models. Metrika, 68, 365-390.

Lee, S. and Lee, T. (2011). Value-at-risk forecasting based on Gaussian mixture ARMA-GARCH model. Journal of Statistical Computation and Simulation, 81, 1131-1144.

Lee, T. and Lee, S. (2009). Normal mixture quasi-maximum likelihood estimator for GARCH models. Scandinavian Journal of Statistics, 36, 157-170.

Nelson, R. (1999). An introduction to copulas, Springer, New York.

Patton, A. J. (2006a). Estimation of multivariate models for time series of possibly different lengths. Journal of Applied Econometrics, 21, 147-173.

Patton, A. J. (2006b). Modeling asymmetric exchange rate dependence. International Economic Review, 47, 527-556.

Rockinger, M. and Jondeau, E. (2006). Modeling the dynamics of conditional dependency between financial series. In Multi-moment Asset Allocation and Pricing Models, edited by Emmanuel Jurzenco and Betrand Maillet, Wiley, West Sussex, 195-222.

Woodward, W. A., Parr, W. C., Schucany, W. R. and Lindsay, H. (1984). A comparison of minimum distance and maximum likelihood estimation of a mixture proportion. Journal of the American Statistical Association, 79, 590-598. 